\title{
A NEW TOPOLOGY ON $B^{*}$-ALGEBRAS ARISING FROM THE ARENS PRODUCTS
}

\author{
EDITH A. McCHAREN
}

\begin{abstract}
A locally convex topology $\mu$ is defined on a Banach algebra $A$. This topology arises naturally from considerations of the Arens products on the second conjugate space $A^{* *}$ of $A$. The main result states that if $A$ is a $B^{*}$-algebra on which the mapping $(a, b) \rightarrow a b$ is $\mu$-continuous for $\|a\| \leqq 1$, then the completion of $A$ with respect to the uniformity generated by $\mu$ is linearly isomorphic to $A^{* *}$. An example is included which shows that this continuity condition does not hold in general as announced by P. C. Shields.
\end{abstract}

Properties of the Arens products on the second conjugate space of a Banach algebra have been widely investigated. In attempting to extend the Arens products to locally convex completions of certain algebras, a particular locally convex topology $\mu$ came to our attention.

In $\S 1$ we define this topology on a Banach algebra $A$ and investigate some of its properties when extended to $A^{* *}$. In $\S 2$ we show that if $A$ is a certain type of $B^{*}$-algebra, then the completion of $A$ with respect to $\mu$ is isomorphic to the second conjugate space of $A$ under the extended $\mu$ topology: Thus, in particular, this completion is an algebra under either Arens product. In $\S 3$ we discuss a topology defined on $W^{*}$-algebras similar to $\mu$ which was announced by P. C. Shields [7] and give a counterexample to one of his assertions.

1. The $\mu$ topology. Let $A$ be a Banach algebra and $A^{*}$ denote its norm dual. For $f \in A^{*}$ and $a \in A$ define two bounded linear functionals, $f \cdot a$ and $a \cdot f$, and a seminorm $p_{f}$ on $A$ in the following way: (i) $f \cdot a(x)=f(a x)$; (ii) $a \cdot f(x)=f(x a)$; and (iii) $p_{f}(x)=\max \{\|f \cdot x\|,\|x \cdot f\|\}$. The $\mu$ topology on $A$ is the locally convex topology determined by the set of seminorms $\left\{p_{f}: f \in A^{*}\right\}$.

Proposition 1. If $A$ has a bounded approximate identity $\left\{e_{\alpha}\right\}$, then the following hold: (1) the $\mu$ topology on $A$ is Hausdorff; (2) the set of $\mu$ continuous linear functionals on $A$, denoted by $(A, \mu)^{*}$, coincides with $A^{*}$;

Received by the editors March 10, 1971.

AMS (MOS) subject classifications (1970). Primary 46LO5; Secondary 46L10, $46 \mathrm{H} 05$.

Key words and phrases. Arens products, locally convex completion of a $B^{*}$-algebra. 
(3) for each $a \in A$ the mappings $x \rightarrow x a$ and $x \rightarrow a x$ on $A$ are $\mu$-continuous; and (4) if, moreover, $A$ has an isometric involution, then it is also $\mu$ continuous.

Proof. (1) If $a \neq 0$, there is $f \in A^{*}$ such that $f(a)>0$ and there exists $e$ in the bounded approximate identity such that $\max \{\|a-a e\|,\|a-e a\|\}<$ $(a) / 2\|f\|$. Therefore, $\max \{\|f \cdot a\|,\|a \cdot f\|\} \geqq f(a) / 2>0$ and so $\mu$ is Hausdorff. (2) follows from the Mackey-Arens theorem and the following inequality: $|f(x)| \leqq \min \left\{\sup \left|f\left(x e_{\alpha}\right)\right|, \sup \left|f\left(e_{\alpha} x\right)\right|\right\} \leqq \max \{\|f \cdot x\|,\|x \cdot f\|\} \leqq$ $\|f\|\|x\|$. (3) is easily verified, and (4) follows readily by defining for $f \in A^{*}$ a bounded linear functional $f^{*}$ on $A$ by $f^{*}(x)=\overline{f\left(x^{*}\right)}$, whence $\left\|f \cdot x^{*}\right\|=$ $\left\|x \cdot f^{*}\right\|$. This completes the proof.

The $\mu$ topology can be extended to $A^{* *}$ where its relationship to the $\sigma\left(A^{* *}, A^{*}\right)$ and $\tau\left(A^{* *}, A^{*}\right)$ topologies is connected with the regularity of the Arens products. For $F \in A^{* *}$ and $f \in A^{*}$ define two linear functionals $F \cdot f$ and $F: f$ on $A$ and a seminorm $P_{f}$ on $A^{* *}$ as follows: (i) $F \cdot f(x)=$ $F(f \cdot x)$; (ii) $F: f(x)=F(x \cdot f)$; and (iii) $P_{f}(F)=\max \{\|F \cdot f\|,\|F: f\|\}$. The $\mu$ topology on $A^{* *}$ is the locally convex topology determined by the set of seminorms $\left\{P_{f}: f \in A^{*}\right\}$.

Arens [1] has defined two products on $A^{* *}$ under which it is a Banach algebra with respect to the usual norm: For $F, G \in A^{* *}$ define $F \cdot G$ by $F \cdot G(f)=F(G \cdot f)$ and $F: G$ by $F: G(f)=F(G: f)$. If $F \cdot G=G: F$ for all $F$, $G \in A^{* *}$ the Arens products on $A^{* *}$ are said to be regular.

PROPOSITION 2. The following are true: (1) if there exists a left identity I for either Arens product, then $\mu$ on $A^{* *}$ is finer than $\sigma\left(A^{* *}, A^{*}\right)$; and

(2) $\mu$ on $A^{* *}$ is coarser than $\tau\left(A^{* *}, A^{*}\right)$ if and only if the Arens products are regular.

Proof. (1) follows since $|F(f)| \leqq\|I\| \max \{\|F \cdot f\|,\|F: f\|\}$. To prove (2) first assume that $\mu$ is coarser than $\tau\left(A^{* *}, A^{*}\right)$. Choose $F, G \in A^{* *}$ such that $\|F\| \leqq 1$. Since the unit ball of $A$ is convex and $\sigma\left(A^{* *}, A^{*}\right)$-dense in the unit ball of $A^{* *}$, it is also $\tau\left(A^{* *}, A^{*}\right)$-dense. Choose a net $\left\{a_{\alpha}\right\}$ in the unit ball of $A$ which converges to $F$ in the $\tau\left(A^{* *}, A^{*}\right)$ topology. Then $\lim \left\|F \cdot f-a_{\alpha} \cdot f\right\|=0$ so that $G \cdot F(f)=\lim G\left(a_{\alpha} \cdot f\right)=\lim (G: f)\left(a_{\alpha}\right)=$ $F: G(f)$.

Conversely, suppose that the Arens products are regular. In $A^{* *}$ let $\left\{F_{\alpha}\right\}$ be a net converging to 0 in the $\tau\left(A^{* *}, A^{*}\right)$ topology and let $f \in A^{*}$. The net $\left\{F_{\alpha}\right\}$ converges to 0 uniformly on every absolutely convex $\sigma\left(A^{*}, A^{* *}\right)$-compact subset of $A^{*}$ and, in particular, on the sets $S \cdot f=\{a \cdot f:\|a\| \leqq 1, a \in A\}$ and $f \cdot S=\{f \cdot a:\|a\| \leqq 1, a \in A\}$ by [5, Theorem 2.1]. Thus

$$
\lim \left(\max \left\{\left\|F_{\alpha}: f\right\|,\left\|F_{\alpha} \cdot f\right\|\right\}\right)=0
$$

and the proof is completed. 
COROLlaRY 1. If $A$ is a Banach algebra with bounded approximate identity $\left\{e_{\alpha}\right\}$ such that $\lim \left\|f \cdot e_{\alpha}-f\right\|=0$ for all $f \in A^{*}$, then $\mu$ on $A^{* *}$ is finer than $\sigma\left(A^{* *}, A^{*}\right)$.

Proof. By Alaoglu's theorem choose $I \in A^{* *}$ to be a $\sigma\left(A^{* *}, A^{*}\right)$-cluster point of the bounded approximate identity. Then $F(f)=\lim F\left(f \cdot e_{\alpha}\right)=$ $I \cdot F(f)$ for each $f \in A^{*}, F \in A^{* *}$.

COROLlaRY 2. If $A$ has a bounded approximate identity and the Arens products are regular, the following hold:

(1) the set of $\mu$-continuous linear functionals on $A^{* *}$ coincides with $A^{*}$; and

(2) the mappings $F \rightarrow F \cdot G$ and $F \rightarrow G \cdot F$ on $A^{* *}$ are $\mu$-continuous.

Proof. Choose $I$ as in Corollary 1. Then $\lim f\left(a e_{\alpha}\right)=f(a)=\lim f\left(e_{\alpha} a\right)$ so that $I \cdot f=f=I: f$ for each $f \in A^{*}$. By regularity $I$ is an identity for both Arens products. (2) is easily verified.

We note here that if $A$ is a $B^{*}$-algebra, then the hypotheses of Corollary 2 are satisfied [4, p. 15]; [2, Theorem 7.1].

2. The continuity condition on $B^{*}$-algebras. In this section we prove the main result:

THEOREM. If $A$ is $a B^{*}$-algebra such that the mapping $(a, b) \rightarrow a b$ on $A$ is $\mu$-continuous for $\|a\| \leqq 1$, then $A^{* *}$ is linearly isomorphic to $(A, \mu)^{\wedge}$, the completion of $A$ with respect to the uniform structure generated by $\mu$.

We freely use the following: Grothendieck's completeness theorem to identify $(A, \mu)^{\wedge}$ with a certain set of linear functionals on $A^{*}$; the fact that $A^{* *}$ is a $W^{*}$-algebra under the Arens product $(F, G) \rightarrow F \cdot G[2]$; and Sakai's representation theorem for $W^{*}$-algebras [6].

Lemma 1. Let $P$ denote the set of positive linear functionals on $A$. Suppose that $\left\{F_{\alpha}\right\}$ is a net of hermitian elements in the unit ball of $A^{* *}$ such that $\lim F_{\alpha} \cdot F_{\alpha}(\theta)=0$ for some $\theta \in P$. If $Q$ denotes the support of $\theta$ in $A^{* *}$, then $\left\{Q \cdot F_{\alpha} \cdot Q\right\}$ converges to zero in the $\mu$ topology.

Proof. The elements of $P$ are exactly the normal positive linear functionals on $A^{* *}[8]$. We shall first show that $\lim Q \cdot F_{\alpha} \cdot Q \cdot Q \cdot F_{\alpha} \cdot Q(\phi)=0$ for all $\phi \in P$. If $A^{* *}$ is represented as a ring of operators on the Hilbert space $H$, then for any $\phi \in P$ there exists a sequence $\left\{x_{i}\right\}$ in $H$ such that $\sum\left\|x_{i}\right\|^{2}<\infty$ and $\phi=\sum\left[x_{i}, x_{i}\right]$, where $\left[x_{i}, x_{i}\right](F)=\left(\left(F x_{i}, x_{i}\right)\right)$ with $(()$, denoting inner product in $H$ [3, p. 51].

For $\varepsilon>0$ choose $N$ such that $\sum_{i=N+1}^{\infty}\left\|x_{i}\right\|^{2}<\varepsilon / 2$. Since

$$
\lim F_{\alpha} \cdot Q \cdot Q \cdot F_{\alpha}(\theta)=\lim \|Q\| F_{\alpha} \cdot F_{\alpha}(\theta)=0
$$


$\left[4\right.$, p. 23], the net $\left\{Q \cdot F_{\alpha} \cdot Q\right\}$ converges to 0 in the strong operator topology $\left[3\right.$, p. 58]. Choosing $\alpha$ sufficiently large, we have that $\left\|Q \cdot F_{\alpha} \cdot Q x_{i}\right\|<\varepsilon / 2 N$ for $i=1, \cdots, N$. Thus $\sum\left\|Q \cdot F_{\alpha} \cdot Q x_{i}\right\|^{2}<\varepsilon$ for sufficiently large $\alpha$.

Since $P$ spans $A^{*}[8]$, for $f \in A^{*}$ there exists a finite number of $\phi_{i} \in P$ such that $|F: f(x)| \leqq \sum\left|F \cdot x\left(\phi_{i}\right)\right|$ for any $F \in A^{* *}$. By the Cauchy-Schwarz inequality we then have $\|F: f\| \leqq \sum\left\|\phi_{i}\right\|^{1 / 2} F \cdot F^{*}\left(\phi_{i}\right)^{1 / 2}$. A similar inequality can be obtained for $\|F \cdot f\|$. The proof is completed by taking $F=$ $Q \cdot F_{\alpha} \cdot Q$

LEMMA 2. Let $A$ be $a B^{*}$-algebra such that the mapping $(a, b) \rightarrow a b$ is $\mu$-continuous for $\|a\| \leqq 1$. Let $S^{\prime}$ denote the unit ball of $A^{* *}$. Then for each $\mu$-neighborhood $U^{\prime}$ of zero in $A^{* *}$ there exists a $\mu$-neighborhood $V$ of zero in $A$ and a $\mu$-neighborhood $W^{\prime}$ of zero in $A^{* *}$ such that $V \cdot\left(W^{\prime} \cap S^{\prime}\right) \subset U^{\prime}$.

Proof. The neighborhood $U^{\prime}$ contains a set of the form

$$
\left\{F \in A^{* *}:\left\|F \cdot f_{n}\right\| \leqq 1,\left\|F: f_{n}\right\| \leqq 1, n=1, \cdots, N\right\} .
$$

Let $U=\frac{1}{2}\left(U^{\prime} \cap A\right)$. Since involution on $A$ is $\mu$-continuous, the mapping $(a, b) \rightarrow a b$ on $A$ for $\|b\| \leqq 1$ is $\mu$-continuous; hence we can choose $\mu$ neighborhoods $V$ and $W$ of 0 in $A$ such that $V \cdot(W \cap S) \subset U$, where $S$ denotes the unit ball of $A$. Since $A$ has a bounded approximate identity, from Proposition 1 we have $A^{*}=(A, \mu)^{*}$ and it follows that $V \cdot(W \cap S)^{\circ \circ} \subset$ $U^{\circ 0}$, where the bipolars are taken in $A^{* *}$.

Let $W^{\prime}=W^{\circ \circ}$ in $A^{* *}$. Noting that $(W \cap S)^{\circ}$ in $A^{*}$ is the $\sigma\left(A^{*}, A\right)$-closed absolutely convex hull of $W^{\circ} \cup S^{\circ}$ and that $W^{\circ}$ and $S^{\circ}$ are $\sigma\left(A^{*}, A\right)$ compact by Alaoglu's theorem, we have that $(W \cap S)^{\circ} \subset W^{\circ}+S^{\circ}$. Thus, $V \cdot\left(W^{\prime} \cap S^{\prime}\right) \subset V \cdot 2(W \cap S)^{\circ} \subset 2 U^{\circ \circ} \subset U^{\prime}$.

It remains to show that $W^{\prime}$ is a $\mu$-neighborhood of zero in $A^{* *}$. If $W$ contains a set of the form $\left\{a \in A:\left\|g_{n} \cdot a\right\| \leqq 1,\left\|a \cdot g_{n}\right\| \leqq 1, n=1, \cdots, N\right\}$, then $W^{\prime}$ contains the set $\left\{F \in A^{* *}:\left\|F \cdot g_{n}\right\| \leqq \frac{1}{2},\left\|F: g_{n}\right\| \leqq \frac{1}{2}\right\}$. For each such $F$ there exists a net $\left\{a_{\alpha}\right\}$ in $A$ such that $F$ is its $\sigma\left(A^{* *}, A^{*}\right)$-limit. Since the Arens products are regular in $A^{* *}$, for each $x$ in $A$ we have that $x \cdot F$ and $x: F$ are the $\sigma\left(A^{* *}, A^{*}\right)$-limits of the nets $\left\{x a_{\alpha}\right\}$ and $\left\{a_{\alpha} x\right\}$ respectively [1]. Therefore, $\max \left\{\left\|a_{\alpha} \cdot g_{n}\right\|,\left\|g_{n} \cdot a_{\alpha}\right\|\right\} \leqq 1$ for sufficiently large $\alpha ;$ thus $F \in W^{\prime}=W^{\circ 0}$ which is $\sigma\left(A^{* *}, A^{*}\right)$-closed.

LemMA 3. If $A$ is a $B^{*}$-algebra and if a net $\left\{a_{\alpha}\right\}$ in $A$ converges to $T \in(A, \mu) \wedge$ in the completion topology, then

$$
|T(f)| \leqq \sup \{|T(f \cdot x)|:\|x\| \leqq 1, x \in A\}=\lim \left\|a_{\alpha} \cdot f\right\|<\infty
$$

for each $f \in A^{*}$.

Proof. Let $\left\{x_{n}\right\}$ be a sequence in the unit ball of $A$ which converges to 0 in norm. Then the sequence $\left\{f \cdot x_{n}\right\}$ is contained in the polar of the 
$\mu$-neighborhood $U=\{x \in A:\|f \cdot x\| \leqq 1,\|x \cdot f\| \leqq 1\}$. Since this sequence certainly converges to 0 in the $\sigma\left(A^{*}, A\right)$ topology, $\lim T\left(f \cdot x_{n}\right)=0$. Thus $T \cdot f$ defined on $A$ by $T \cdot f(x)=T(f \cdot x)$ is norm-continuous.

For $\varepsilon>0$ and $U$ as above $\varepsilon U^{\circ \circ} \cap(A, \mu)^{\wedge}$ in $A^{*^{\prime}}$, the set of linear functionals on $A^{*}$, is a neighborhood of 0 in the completion topology. Thus $T-a_{\alpha}$ is in this neighborhood for sufficiently large $\alpha$. Since $f \cdot x \in U^{\circ}$ for each $x \in A$ with $\|x\| \leqq 1$, it follows that $\|T \cdot f\|=\lim \left\|a_{\alpha} \cdot f\right\|$.

If $I$ is a $\sigma\left(A^{* *}, A^{*}\right)$-cluster point of a bounded approximate identity $\left\{e_{\alpha}\right\}$ for $A$, then $I: f=f$ for all $f \in A^{*}$ (see Corollary 2 ), and thus by regularity we have that $f$ is a $\sigma\left(A^{*}, A^{* *}\right)$-cluster point of the net $\left\{f \cdot e_{\alpha}\right\}$ [1]. Hence $|T(f)| \leqq \sup \left|T\left(f \cdot e_{\alpha}\right)\right| \leqq\|T \cdot f\|$. This completes the lemma.

ProOf OF THE TheOREM. For $F \in A^{* *}$ choose a net in $A$ for which $F$ is its $\tau\left(A^{* *}, A^{*}\right)$-limit. Since the Arens products are regular, $F$ is also the $\mu$ limit of this net by Proposition 2. Since the net in $A$ is $\mu$-Cauchy, it follows that $F \in(A, \mu)^{\wedge}$.

To show that $(A, \mu)^{\wedge}$ is contained in $A^{* *}$, it suffices to show that if $\left\{\theta_{n}\right\}$ is a sequence of positive linear functionals in $A^{*}$ such that $\left\|\theta_{n}\right\| \leqq 1 / 2^{n}$ then $\lim T\left(\theta_{n}\right)=0$ for $T \in(A, \mu)^{\wedge}$ [8].

Let $\theta=\sum \theta_{n}$; then $\theta$ is a normal positive linear functional on $A^{* *}$ and $0 \leqq F^{*} \cdot F\left(\theta_{n}\right) \leqq F^{*} \cdot F(\theta)$ for all $F$ in $A^{* *}$. Choose a sequence $\left\{G_{n}\right\}$ in $A^{* *}$ such that $0 \leqq G_{n} \leqq 1$ and $F\left(\theta_{n}\right)=G_{n} \cdot F \cdot G_{n}(\theta)$ for all $F$ in $A^{* *}[3$, p. 63]; then if $Q$ denotes the support of $\theta$, we have that $0 \leqq Q \cdot G_{n} \cdot Q \cdot Q \cdot G_{n} \cdot Q(\theta)=$ $Q\left(\theta_{n}\right) \leqq\left\|\theta_{n}\right\|$. Consequently, the sequence $\left\{Q \cdot G_{n} \cdot Q\right\}$ converges to 0 in the $\mu$ topology by Lemma 1 .

Now let $\varepsilon>0$ and consider the $\mu$-neighborhood $U^{\prime}=\left\{F \in A^{* *}:\|F \cdot \theta\| \leqq\right.$ $\varepsilon / 2,\|F: \theta\| \leqq \varepsilon / 2\}$ in $A^{* *}$. By Lemma 2 there exists a $\mu$-neighborhood $V$ of 0 in $A$ and a $\mu$-neighborhood $W^{\prime}$ of 0 in $A^{* *}$ such that $V \cdot\left(W^{\prime} \cap S^{\prime}\right) \subset U^{\prime}$, where $S^{\prime}$ denotes the unit ball of $A^{* *}$.

Let $\left\{a_{\alpha}\right\}$ be a $\mu$-Cauchy net in $A$ converging to $T \in(A, \mu)^{\wedge}$. Fix $a$ in this net such that $a-a_{\alpha} \in V$ for sufficiently large $\alpha$. Choose $N$ such that $\left\|\theta_{n}\right\|<\varepsilon / 2(\|a\|+1)$ for $n>N$, and $M$ such that $Q \cdot G_{n} \cdot Q \in W^{\prime}$ for $n>M$. Let $F=\left(a_{\alpha}-a\right) \cdot Q \cdot G_{n} \cdot Q$ for $n>N+M$ and $\alpha$ sufficiently large so that $F \in V \cdot\left(W^{\prime} \cap S^{\prime}\right) \subset U^{\prime}$. Then $\left\|\left(Q \cdot G_{n} \cdot Q\right):(F \cdot \theta)\right\| \leqq \varepsilon / 2$. From the relations on $Q$ and $G_{n}$ and $[3$, p. 59] we have for $x \in A$ that

$$
\begin{aligned}
\left(Q \cdot G_{n} \cdot Q\right):(F \cdot \theta)(x) & =Q \cdot x \cdot\left(a_{\alpha}-a\right) \cdot Q\left(\theta_{n}\right) \\
& =\left[x \cdot\left(a_{\alpha}-a\right)\right]\left(\theta_{n}\right)=\left(a_{\alpha}-a\right) \cdot \theta_{n}(x) .
\end{aligned}
$$

Therefore, $\left\|a_{\alpha} \cdot \theta_{n}\right\|<\varepsilon$ for $n>N+M$ and sufficiently large $\alpha$. Thus by Lemma 3 we have that $\left|T\left(\theta_{n}\right)\right|$ is bounded by the limit with $\alpha$ of the net $\left\{\left\|a_{x} \cdot \theta_{n}\right\|\right\}$, which is at most $\varepsilon$ for $n$ large. 
Therefore, $A^{* *}$ and $(A, \mu)$ are equal as sets. That the $\mu$ topology and the completion topology are the same is essentially proved in Lemma 2.

3. The continuity condition on $W^{*}$-algebras. A topology on $W^{*}$-algebras similar to the extended $\mu$ topology was announced by P. C. Shields [7] for which he claimed that the mapping $(a, b) \rightarrow a b$ (for $\|a\| \leqq 1$ ) on an arbitrary $W^{*}$-algebra is continuous. In this section we give a counterexample to his assertion.

Let $M$ be a $W^{*}$-algebra with predual $F$. Conventionally we consider $F$ as a set of linear functionals on $M$ and so use the notation established previously. Shields' topology is determined by the seminorms $x \rightarrow$ $\max \{\|f \cdot x\|,\|x \cdot f\|\}$ for all $f \in F$. We shall also refer to this topology as $\mu$. If $M$ is commutative, the mapping $(a, b) \rightarrow a b$ for $\|a\| \leqq 1$ is $\mu$-continuous: It follows directly from the identity $a b-a_{\alpha} b_{\alpha}=\left(a-a_{\alpha}\right) b+a_{\alpha}\left(b-b_{\alpha}\right)$. The next proposition establishes the means by which we construct a counterexample for the general case.

Proposition 3. Suppose there exist $\varepsilon>0$ and a positive linear functional $\theta$ in $F$ which satisfy the following property: For every $\delta>0$ and for every positive linear functional $\phi$ in $F$ there exist equivalent projections $p$ and $q$ in $M$ such that $\theta(p)>\varepsilon$ and $\phi(q)<\delta$. Then the mapping $(a, b) \rightarrow a b$ on $M$ is not $\mu$-continuous for $\|a\| \leqq 1$.

Proof. Let $U$ be the $\mu$-neighborhood $\{x \in M:\|\theta \cdot x\| \leqq \varepsilon,\|x \cdot \theta\| \leqq \varepsilon\}$; let $V=\left\{x \in M:\left\|\psi_{k} \cdot x\right\| \leqq \sigma,\left\|x \cdot \psi_{k}\right\| \leqq \sigma, k=1, \cdots, K\right\}$ and $W=\{x \in M$ : $\left.\left\|\phi_{n} \cdot x\right\| \leqq \eta,\left\|x \cdot \phi_{n}\right\| \leqq \eta, n=1, \cdots, N\right\}$ be any two basic $\mu$-neighborhoods of 0 . Choose $\lambda>0$ such that $\lambda \leqq \min \left\{1, \sigma /\left(1+\sum\left\|\psi_{k}\right\|\right)\right\}$. Let $\phi=\sum \phi_{n}$ and $\delta=\left[\lambda \eta /\left(1+\sum\left\|\phi_{n}\right\|^{1 / 2}\right)\right]^{2}$. Then by hypothesis for $\phi$ and $\delta$ there exist projections $p, q \in M$ and $v \in M$ such that $v v^{*}=p, v^{*} v=q, \theta(p)>\varepsilon$ and $\phi_{n}(q) \leqq \phi(q)<\delta$ for all $n$.

Since $\|v\|=1$ so that $\max \left\{\left\|\lambda v \cdot \psi_{k}\right\|_{1},\left\|\psi_{k} \cdot \lambda v\right\|\right\} \leqq \sigma$ for all $k$, we see that $\lambda v \in V \cap S$, where $S$ denotes the unit ball of $M$. Furthermore, as a consequence of the Cauchy-Schwarz inequality we have that

$$
\max \left\{\left\|(1 / \lambda) q \cdot \phi_{n}\right\|,\left\|\phi_{n} \cdot(1 / \lambda) q\right\|\right\} \leqq(1 / \lambda)\left\|\phi_{n}\right\|^{1 / 2} \phi_{n}(q)^{1 / 2} \leqq \eta,
$$

for all $n$; hence $(1 / \lambda) q \in W$ and $v q \in(V \cap S) \cdot W$.

Noting that $q v^{*}=v^{*}$, we have $\|\theta \cdot v q\| \geqq \theta\left(v q v^{*}\right)=\theta(p)>\varepsilon$. Thus the mapping $(a, b) \rightarrow a b$ is not $\mu$-continuous for $\|a\| \leqq 1$ at the point $a=0=b$.

COROLlaRY. If $M$ is a $W^{*}$-algebra containing an infinite number of nonzero equivalent projections, then the mapping $(a, b) \rightarrow a b$ on $M$ is not $\mu$ continuous for $\|a\| \leqq 1$.

ProOF. Let $\left\{p_{n}\right\}$ be an infinite set of nonzero equivalent orthogonal projections. Since $F$ is spanned by the normal positive linear functionals 
on $M$, there is one such functional $\theta$ for which $\theta\left(p_{1}\right)>0$. For any positive linear functional $\phi \in F$ and $\delta>0$ there exists $N$ such that $\phi\left(p_{N}\right)<\delta$. If this were not true, then by choosing $n$ such that $n \delta>\|\phi\|$ we have $n \delta \leqq$ $\phi\left(\sum_{i=1}^{n} p_{i}\right) \leqq\|\phi\|$ since $\phi$ is linear and the sum of these projections is a projection, a contradiction. The conclusion now follows from the proposition with $\varepsilon=\theta\left(p_{1}\right)$.

Thus, if $H$ is an infinite dimensional Hilbert space, the set of all bounded linear functionals on $H$ clearly satisfies the hypothesis of the above Corollary.

\section{REFERENCES}

1. R. F. Arens, The adjoint of a bilinear operation, Proc. Amer. Math. Soc. 2 (1951), 839-848. MR 13, 659.

2. P. Civin and B. Yood, The second conjugate space of a Banach algebra as an algebra, Pacific J. Math. 11 (1961), 847-870. MR 26 \#622.

3. J. Dixmier, Les algèbres d'opérateurs dans l'espace Hilbertiens, 2ième éd., Cahiers Scientifiques, fasc. 25, Gauthier-Villars, Paris, 1969.

4. - Les $C^{*}$-algèbres et leurs représentations, 2ième éd., Cahiers Scientifiques, fasc. 29, Gauthier-Villars, Paris, 1969. MR 39 \#7442.

5. J. Hennefeld, A note on the Arens products, Pacific J. Math. 26 (1968), 115-119. MR 37 \#6755.

6. S. Sakai, A characterization of $W^{*}$-algebras, Pacific J. Math. 6 (1956), 763-773. MR 18, 811.

7. P. C. Shields, A new topology for von Neumann algebras, Bull. Amer. Math. Soc. 65 (1959), 267-269. MR 21 \#5917.

8. Z. Takeda, Conjugate spaces of operator algebras, Proc. Japan Acad. 30 (1954), 90-95. MR 16, 146.

Department of Mathematics, Northern Illinois University, DeKalb, Illinois 60115 